\title{
A novel quantitative evaluation approach based on neutrality theory
}

\author{
Dai $\mathrm{CHEN}^{1, \mathrm{a}^{*}}$, Ligui $\mathrm{KANG}^{1, \mathrm{~b}}$, Hang Cheng ${ }^{1, \mathrm{c}}$, Jianjun Chen ${ }^{1, \mathrm{~d}}$, Qiang $\mathrm{SHAO}^{2, \mathrm{e}}$ \\ ${ }^{1}$ Chongqing Special Equipment Inspection and Research Institute, Chongqing, China \\ ${ }^{2}$ CISIDI Group Co., LTD, Chongqing, China \\ a915156921@qq.com, b420725690@qq.com, ${ }^{\text {c3 } 30126891 @ q q . c o m, ~ d 382474045 @ q q . c o m, ~}$ \\ eshaoqiang_83@sohu.com
}

Keywords: Quantitative evaluation; Analytical hierarchy process (AHP); Difficulty coefficient mechanism Neutrality principle.

\begin{abstract}
As to the respective quantitative evaluation of the professional technical personnel and managers in institution, the evaluation factors and their specific weights has been introduced by the questionnaire investigation and analytical hierarchy process (AHP) in this paper. To improve the rationality and justice, the weight of sub-factor attached to certain factors need to be revised further by the difficulty coefficient mechanism. To be specific, the preference of the employees and the present situation in this institution should be taken into consideration. At last, the weight calculation mechanism has been proposed for the fusion evaluation model based on neutrality principle.
\end{abstract}

\section{Introduction}

The result of examination and evaluation has impact on the distribution of the performance salary, training, engagement of position, etc. of the examined. Reasonable, perfect and effective evaluation system plays a key role for motivating employees. For scientific research institution, the evaluation is one of the core content of management. At present, there exist two problems in quantitative evaluation management for most of the units, one is that the quantitative assessment system is designed with faultiness, the other is lack of sufficient basis theoretical. As is well known, the quantitative analysis methods mainly include expert consulting method and analytic hierarchy process (AHP).

Usually normal AHP must assume independency among criteria, although it is difficult to choose enough independent criteria. Inner dependence method AHP is one technique of solving this kind of problem even in case of criteria have dependency. However, the comparison matrix often does not have enough consistency when AHP or Inner dependence method is used since, for instance, a problem may contain too many criteria for decision making. In these cases, we consider that answers from decision-makers (i.e. components of the comparison matrix) have ambiguity or fuzziness. Nevertheless, these kinds of methods have strong subjectivity with out of the objective attribute of the current situation. In a word, the individual preferences of leaders may affect the judgment results of evaluation.

According to the above problem, a novel quantitative evaluation approach based on the AHP and the difficulty coefficient algorithm with neutrality principle is proposed in this paper. Such benefit is that there are both subjective preference of broad employees, also consider the exist situation. The factors and the corresponding quantification scores are determined through the questionnaire and analysis of objective conditions which improve the management level and the scientific nature. 


\section{Framework of quantitative evaluation system}

The basic principle of quantitative evaluation system is continuity, equity and publicity. The model is based on the weighted method and differentiated by different post. The evaluation system function can be described as follows

$$
\mathrm{P}=\sum_{i=1}^{n} \alpha_{i} A_{i}
$$

Where $P$ denotes total score of employees, $\alpha_{i}$ denotes the weighting of element $i, \mathrm{~A}_{\mathrm{i}}$ denotes the scores in element $i$ of employees. In view of the institution is mainly composed of two posts, namely professional technical and management. According to the characteristics of the posts, the quantitative appraisal system is built respectively in order to reflect the comprehensive efficiency of each post personnel. Around the above principle, primary evaluation factors are designed as shown in table1 for professional personnel and management personnel respectively.

Table 1 Factors of appraisal evaluation system

\begin{tabular}{|c|c|}
\hline post & primary evaluation factors \\
\hline professional technical & $\begin{array}{r}\text { work experience, office life, democratic appraisal, } \\
\text { annual assessment, inspector qualification, } \\
\text { inspection quality, scientific research, academic } \\
\text { participation }\end{array}$ \\
\hline management & $\begin{array}{c}\text { work experience, office life, democratic appraisal, } \\
\text { annual assessment, academic situation, commend } \\
\text { ations situation }\end{array}$ \\
\hline
\end{tabular}

\section{The proposed approach for calculating weight}

Primary factors of evaluation system has been determined above, some primary factors need to determine the secondary factors (such as scientific research and commendations situation), as well as the weight scores of each evaluation factor. AHP was proposed by Saaty T.L. in 1977. The method has been popular and widely used in the field of decision making, since it can reflect humans feelings naturally. A pairwise comparison matrix $\mathrm{C}$ is created from questionnaire of whole employees.

$$
C=\left[\begin{array}{cccccccc}
c_{11} & c_{12} & \cdot & \cdot & \cdot & \cdot & \cdot & c_{18} \\
c_{21} & c_{22} & & \cdot & & . & & c_{28} \\
\cdot & & \cdot & & \cdot & & & \cdot \\
\cdot & \cdot & & \cdot & & \cdot & \cdot & \cdot \\
\cdot & & \cdot & & \cdot & & & \cdot \\
\cdot & . & & . & & . & & \cdot \\
\cdot & & & & . & & . & \cdot \\
c_{81} & . & . & . & \cdot & . & c_{87} & c_{88}
\end{array}\right]=\left[\begin{array}{cccccccc}
1 & 7 / 5 & 7 / 5 & 7 & 7 / 5 & 7 / 9 & 7 / 3 & 7 / 6 \\
5 / 7 & 1 & 4 / 3 & 5 & 1 & 5 / 9 & 2 & 1 \\
5 / 7 & 3 / 4 & 1 & 4 & 3 / 4 & 1 / 3 & 2 & 1 \\
1 / 7 & 1 / 5 & 1 / 4 & 1 & 1 / 4 & 1 / 9 & 1 / 3 & 1 / 5 \\
5 / 7 & 1 & 4 / 3 & 4 & 1 & 4 / 9 & 5 / 3 & 4 / 5 \\
9 / 7 & 9 / 5 & 3 & 9 & 9 / 4 & 1 & 3 & 9 / 5 \\
3 / 7 & 1 / 2 & 1 / 2 & 3 & 3 / 5 & 1 / 3 & 1 & 1 / 2 \\
6 / 7 & 1 & 1 & 5 & 5 / 4 & 5 / 9 & 2 & 1
\end{array}\right]
$$

In addition, for diagonal elements $c_{\mathrm{ij}}=1$ (when $\mathrm{i}=\mathrm{j}$ ). The lower triangular components and diagonal elements are $c_{i j}=1 / c_{j i}$. The order in $C$ from left to right is in accordance with factors contained in 
table1. Through the matrix operations, the maximum eigenvalue of $C$ is $\lambda_{\max }=7.9945$. The result is

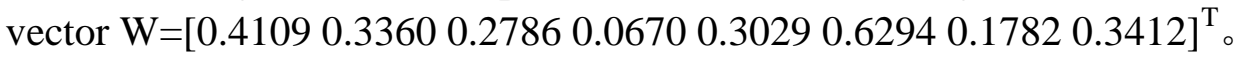

Since components of the comparison matrix are obtained by comparisons between two elements, coherent consistency is not guaranteed. In AHP, the consistency of the comparison matrix C is measured by the following consistency ratio CR. It should be noted that $\mathrm{CR} \geq 0$ holds. And if the value of $\mathrm{CR}$ becomes smaller, then the degree of consistency becomes higher, and vice versa. The comparison matrix is consistent if the $\mathrm{CR}<0.1$ holds.

$$
C R=\frac{C I}{R I}=\frac{\lambda_{\mathrm{m} \mathrm{ax}}-n}{(n-1 \not R I}=\frac{7.9945}{(-8 \times 1)} \stackrel{8}{=} \underset{0.4}{0.00} 0 \propto 6
$$

That is to say it is passed the consistency inspection. Because the quantitative evaluation system is designed as the form of scoring, the elements in vector $\mathrm{W}$ has been timed twenty and taken the integer portion. It can be obtained the score of elements $W_{1}=\left[\begin{array}{lllllll}8 & 6 & 5 & 1 & 6 & 12 & 3\end{array}\right]^{\mathrm{T}}$ 。

By analyzing further, it can be known that multiple secondary factors are contained in some primary factors, such as scientific research situation include thesis, patents, standards, project and so on. Even, in such a paper categories, there are divided into several levels including SCI indexed papers, EI indexed papers and papers in core journals according to the classification standard from the Chinese academy of sciences. For the same kinds of quantitative attributes, difficulty coefficient algorithm is proposed to calculate the scores of secondary factors based on the investigation and analysis of present situation. According to statistics of existing achievements, the project which has largest number is the lowest difficulty coefficient, and the difficulty coefficient is set to one for basis, then the difficulty coefficient calculation formula of remainder projects can be described as follows

$$
\Theta_{j}=\frac{S_{r_{e f}}}{\kappa_{j} S_{j}} \quad j=1,2, \cdots, \mathrm{n}
$$

Where $S_{\text {ref }}$ denotes the ratio of lowest difficulty coefficient, $\kappa_{\mathrm{j}}$ denotes the correction factor. When $S_{\mathrm{j}}=0$, it is indicated that there is a large difficulty in actual and it need to be determined the score separately for such item. For other items, the score is equal to the difficulty coefficient multiplied by the basic score. Take thesis project as example, the specific calculation method based on proposed difficulty coefficient approach is introduced detailed. According to the classification standard from Chinese academy of sciences, the paper is classified as SCI indexed papers, EI indexed papers, papers in core journals and papers in non-core ordinary journals. As is well-known, the paper in non-core ordinary journal is the least difficult difficulty which is set to one for basis. According to the statistical data available, the ratio of SCI indexed papers is 0, ratio of EI indexed papers is 3.3\%, ratio of papers in core journals is $4.2 \%$, ratio of papers in non-core ordinary journals is $92.5 \%$. However, with reference to the normal scientific laws, ratio relationship of each level should be $1: 3: 6: 18$, so the correction coefficient of EI indexed papers and papers in core journal is 1.7 and 1.6 respectively. By the difficulty coefficient calculation formula, the score of EI indexed papers and papers in core journal are 15 and 12 respectively.

\section{Summary}

Quantitative evaluation problem is very complicated which need to continue to research and explore continuously. A perfect quantitative evaluation system can not be completed in a short time, while it may be a continuous feedback and correction process. A novel weight calculation mechanism has 
been proposed for the fusion evaluation model based on neutrality principle which can stimulate the enthusiasm in work and stabilize the mentality of the employees to some certain.

\section{Acknowledgement}

The authors would like to express their sincere thanks to the anonymous reviewers for their valuable suggestions and comments to improve this paper. This work is supported by science and technology planning project of General Administration of Quality Supervision, Inspection and Quarantine of the People's Republic of China (No.2014QK260).

\section{References}

[1] Xiaoqin. AHP-based evaluation model for service composition[J]. Computer science, 2006, 3983:756-766.

[2] Jia Peipei, Zhang Tai. Based on AHP quantitative assessment of tourism impact[J]. Compute and information science, 2011, 243: 205-212.

[3] MA Jian, FAN Zhiping, HUANG Lihua. A subjective and objective integrated approach to determine attribute weights[J]. European Journal ofOperational Research, 1999, 112(2): 397-404.

[4] ZHAO Yong, HUANG Dingxuan, TAN Jianxin . Case and confidence-level-based significance ordering of multiple factors[J]. Journal of Southwest Jiaotong University, 2003, 38 (1):102-105.

[5]S. Ohnishi, T. Yamanoi, H. Imai.

A Fuzzy Weight Representation for Inner Dependence AHP[J]. Journal of Advanced Computationa 1 Intelligence and Intelligent Informatics, Vol.15, No.3, 2011, pp: 329-335.

[6]M.K. Chen, S. c. Wang.

The critical factors of success for information service industry in developing international market: Using analytic hierarchy process (AHP) approach[J]. Expert Systems with Applications, vol. 37 pp .694-704,2010.

[7]O. López-Ortega, M.-A. Rosales.

An agent-oriented decision support system combining fuzzy clustering and the AHP $[J]$. Expert Sys tems with Applications, vol. 38, pp. 8275-8284,2011.

[8]H. Jung.A fuzzy AHP-GP approach for integrated production-planning considering manufacturing partners[J]. Expert Systems with Applications, vol. 38, pp. 5833-5840,2011.

[9]Ludovic-Alexandre Vidal, Franck MarIe, Jean-Claude Bocquet.Using a Delphi process and the An alytic Hierarchy Process (AHP) to evaluate the complexity of projects[J]. Expert Systems with App lications, vol. 38, pp. 5388-5405,2011.

[10]Du dong, Pang Qinghua. Modern comprehensive evaluation method and case selection[M]. Tsinghua University Press, 2008. 